SCIENTIFIC CORRESPONDENCE

\title{
Macular hole size as a prognostic factor in macular hole surgery
}

\author{
S Ullrich, C Haritoglou, C Gass, M Schaumberger, M W Ulbig, A Kampik
}

Br J Ophthalmol 2002;86:390-393

Background/aim: In 1991 there was a series of successful closures of a macular hole after vitrectomy and membrane peeling. Today this technique has become a standard procedure. The aim of this study was to evaluate the role of optical coherence tomography in diagnosing and staging, as well as in predicting, the functional and anatomical outcome after macular hole surgery.

Method: In a prospective study 94 consecutive patients (20 male, 74 female) with a mean age of 67.6 (SD 6.0) years and a macular hole stage II $(n=8), I I I(n=72)$, and IV $(n=14)$ according to the classification by Gass were examined with optical coherence tomography (OCT) before pars plana vitrectomy. Macular hole diameters were determined at the level of the retinal pigment epithelium (base diameter) and at the minimal extent of the hole (minimum diameter). Calculated hole form factor (HFF) was correlated with the postoperative anatomical success rate and best corrected visual acuity. The duration of symptoms was correlated with base and minimum diameter of the macular hole.

Results: In eyes without anatomical closure of the macular hole after one surgical approach (13/94) the base diameter ( $\mathrm{p} 1$ ) and the minimum diameter (p2) were significantly larger than in cases with immediate postsurgical closure (pl $=0.003 ; p 2=0.028$ ). There was a significant negative correlation between both the base and the minimum diameter of the hole and the postoperative visual function (p1 $=0.016 ;$ p2 $=0.002$ ). In all patients with HFF $>0.9$ the macular hole was closed following one surgical procedure, whereas in eyes with HFF $<0.5$ anatomical success rate was $67 \%$. Better postoperative visual outcome correlated with higher HFF $(p=0.050)$. There was no significant correlation between the duration of symptoms and base or minimum diameters $(\mathrm{p} 1=0.053$; $\mathrm{p} 2=0.164)$, respectively.

Conclusion: Preoperative measurement of macular hole size with OCT can provide a prognostic factor for postoperative visual outcome and anatomical success rate of macular hole surgery. The duration of symptoms did not correlate with the diameters measured. Base and minimum diameters especially seem to be of predictive value in macular hole surgery.

$\mathrm{T}$ he first clinical description of a macular hole was published by Henry Noyes in 1871.' Since then our understanding of development and pathogenesis of macular holes has much improved. However, it took more than 100 years, until Kelly and Wendell reported the first successful closure of a series of macular holes by pars plana vitrectomy and membrane peeling in 1991. ${ }^{2}$ Today this procedure has become a standard to improve or at least stabilise visual acuity of affected eyes. Postoperative anatomical success rate varies between $86 \%$ and $95 \%$ in the literature and all authors report an improvement in visual acuity in the majority of vitrectomised eyes. ${ }^{3-6}$

Optical coherence tomography (OCT) is a recently introduced diagnostic tool for high resolution, cross sectional imaging of the posterior and anterior segment of the eye with a resolution of approximately $10 \mu \mathrm{m}^{78}$

The aim of this study was to evaluate the role of preoperative OCT measurements and its impact on diagnosing and staging and its predictive value for postoperative anatomical and functional outcome of idiopathic macular holes.

\section{METHOD}

In a prospective study, 94 consecutive patients with an idiopathic full thickness macular hole were evaluated. Patients with other underlying macular diseases such as macular degeneration or diabetic retinopathy were not included. Staging of the macular hole was performed biomicroscopically with a slit lamp examination according to the Gass classification ${ }^{9}$ and by OCT examination. The staging results of these two approaches were compared.

Visual acuity was measured preoperatively, at 6 and 12 weeks and at 6 and 12 months, postoperatively. For statistical analysis only the best corrected postoperative visual acuity achieved by each patient at one of these visits was used.

Surgery consisted of standard three port pars plana vitrectomy, peeling of the epiretinal membranes if present and the inner limiting membrane with subsequent instillation of a gas tamponade $\left(\mathrm{C}_{2} \mathrm{~F}_{6}\right) \cdot{ }^{10}{ }^{11}$ No substances such as indocyanine green (ICG) or growth factors such as transforming growth factor $\beta$ (TGF- $\beta$ ) were used. After surgery patients were asked to maintain a prone position for at least 5 days. Postoperative anatomical success was defined as flattening of the hole with no subretinal fluid.

Two macular hole diameters were defined using OCT. The first diameter was measured at the level of the retinal pigment epithelium (base diameter), and the second at the minimal extent of the hole (minimum diameter). The hole form factor (HFF) was calculated according to Puliafito and colleagues ${ }^{12}$ (Fig 1). The HFF and the diameters measured were correlated with the best corrected postoperative visual acuity and visual improvement.

According to the reported duration of symptoms the patients were divided into four groups: A: 0-3 months, B: 3-6 months, C: 6-12 months, and D more than 12 months. Base and minimum hole diameter measured with OCT were then correlated with the duration of symptoms.

Statistical tests were performed using spss for Windows 9.0.0.

\section{RESULTS}

A total of 94 eyes of 94 consecutive patients with idiopathic macular holes were included in the study. There were 74 (78.7\%) women and $20(21.3 \%)$ men, with an average age of 


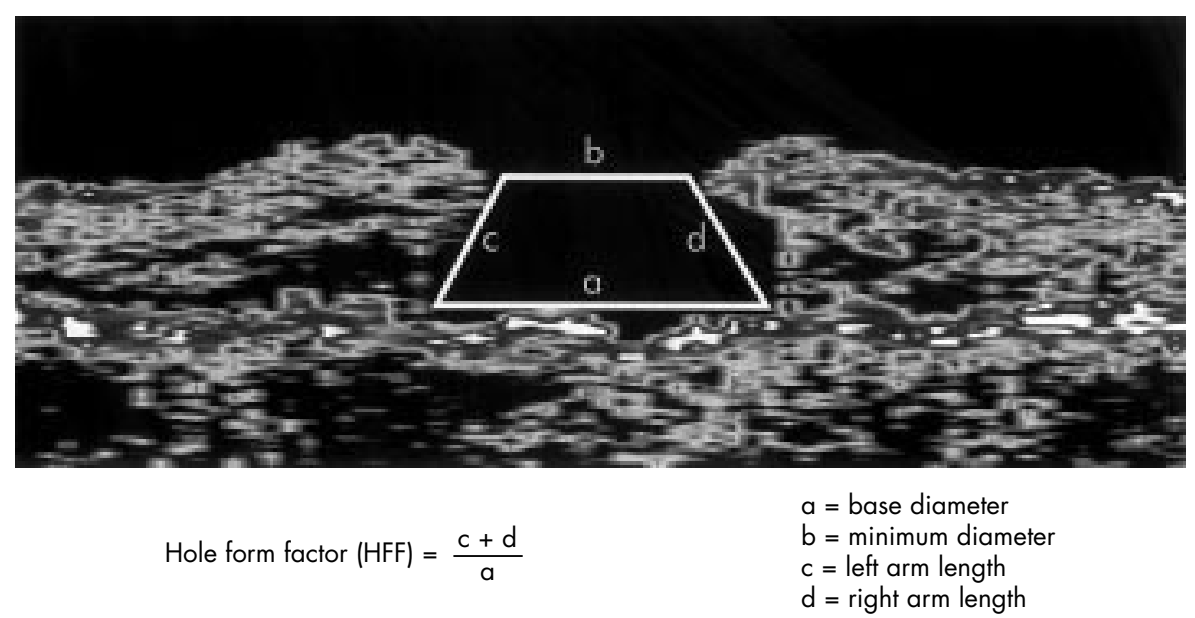

Figure 1 Optical coherence tomography of a macular hole.

68 years (50-80 years, median 67 years). The postoperative follow up ranged from 6 weeks to 12 months. Mean follow up time was 7.1 months. At this time 38 patients were pseudophakic and 56 were phakic. None of the 56 patients with their crystalline lens revealed lens opacities which clinically interfered with visual acuity. At 1 year 46 of 60 patients $(77 \%)$ attained a visual acuity greater than or equal to $20 / 50$. Furthermore, no changes being suggestive for phototoxicity were noted postoperatively. There were two cases of postoperative retinal detachment without affecting the macular area.

On slit lamp examination a macular hole stage II (according to the classification proposed by Gass) was diagnosed in eight eyes, stage III was diagnosed in 71 eyes, and stage IV in 14 eyes. Using OCT the macular hole was staged grade II in four eyes (three eyes consistent with slit lamp examination), grade III in 29 eyes ( 23 eyes consistent with slit lamp examination), and grade IV in 40 eyes (six eyes consistent with slit lamp examination). In 20 eyes a differentiation between stage III and IV could not be made using OCT.

A significant negative correlation between both the base (p1) and the minimum diameter (p2) of the hole and postoperative visual acuity was found (Spearman rank correlation: $\mathrm{pl}=0.016$ and $\mathrm{p} 2=0.002$ respectively; Fig $2 \mathrm{~A}, \mathrm{~B})$. The better postoperative visual outcome was positively correlated with a higher HFF value (Spearman rank correlation: $\mathrm{p}=0.050$; Fig 3).

No correlation could be found between postoperative gain in lines and HFF $(\mathrm{p}=0.76)$ nor with the base and minimum diameter $(\mathrm{pl}=0.19 ; \mathrm{p} 2=0.071)$.

In eyes without anatomical closure after the first surgical procedure (13 of 94$)$ the base diameter $(\mathrm{p}=0.003)$ and the minimum diameter $(\mathrm{p}=0.028)$ of the hole was significantly larger than in cases with immediate closure (Fig 4).

In all patients with HFF $>0.9(10 / 10)$ the macular hole was closed following one surgical approach whereas in eyes with HFF $\leqslant 0.5$ anatomical success rate after one operation was only $67 \%(4 / 6)$. With respect to the correlation coefficient, $r$, the minimum diameter measured with OCT seems to be a better predictor than the HFF (minimum diameter $r=-0.312$; HFF $r=0.203$ ).

There was no significant correlation between the duration of symptoms and the diameters measured (base: $p=0.053$; minimum: $\mathrm{p}=0.164$; Fig 5 )

\section{DISCUSSION}

The aim of this study was to evaluate the role of a preoperative optical coherence tomography (OCT) measurement in diagnosing and staging of macular holes and its predictive value for the anatomical and functional outcome after macular hole surgery.
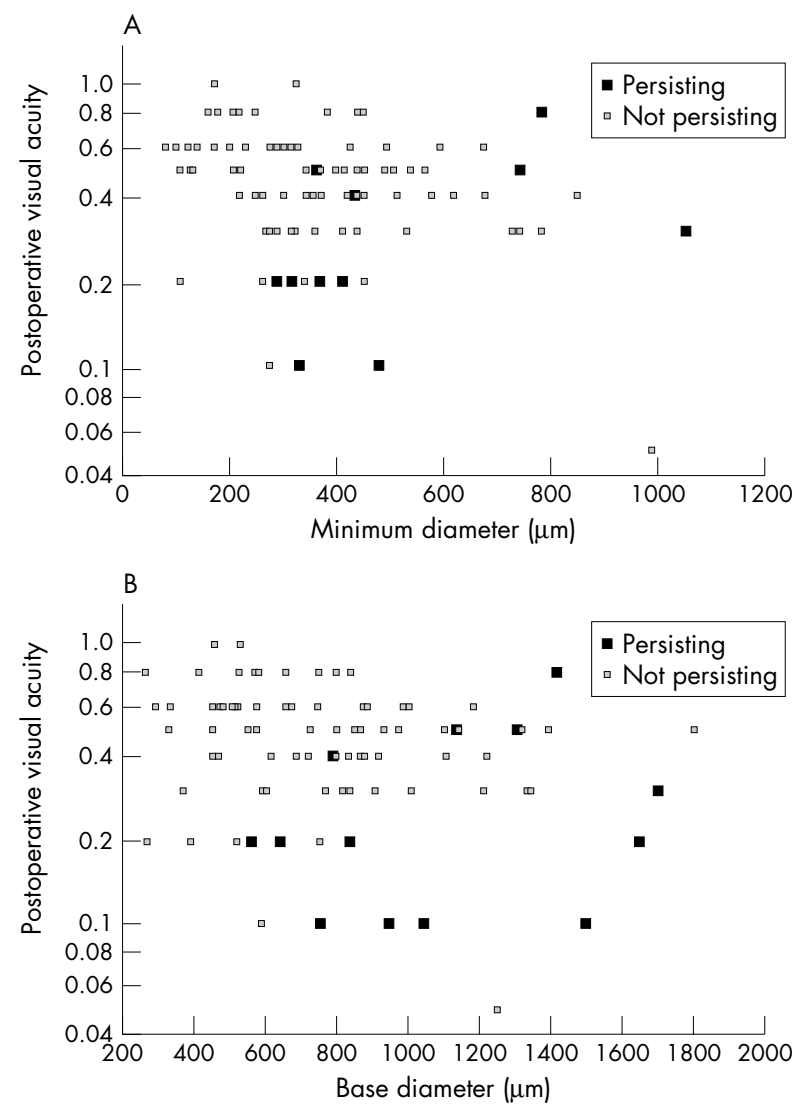

Figure 2 (A) Correlation of the minimum diameter of the macular hole and best corrected postoperative visual acuity. (B) Correlation of the base diameter of the macular hole and best corrected postoperative visual acuity.

The diameter of the macular hole measured by OCT at the level of the retinal pigment epithelium and the minimum diameter seem to provide a prognostic factor for postoperative visual outcome and anatomical success of macular hole surgery. The base diameter, which can only be measured by OCT, seems to have a better prognostic value compared to slit lamp examination as it reflects the real size of the retinal lesion. Similar results have been published by Freeman and coworkers, who found that a macular hole with a small diameter was associated with better functional outcome. ${ }^{13}$ The reason for this might be that a small hole diameter indicates a better preserved macula. Differences of OCT and clinical 


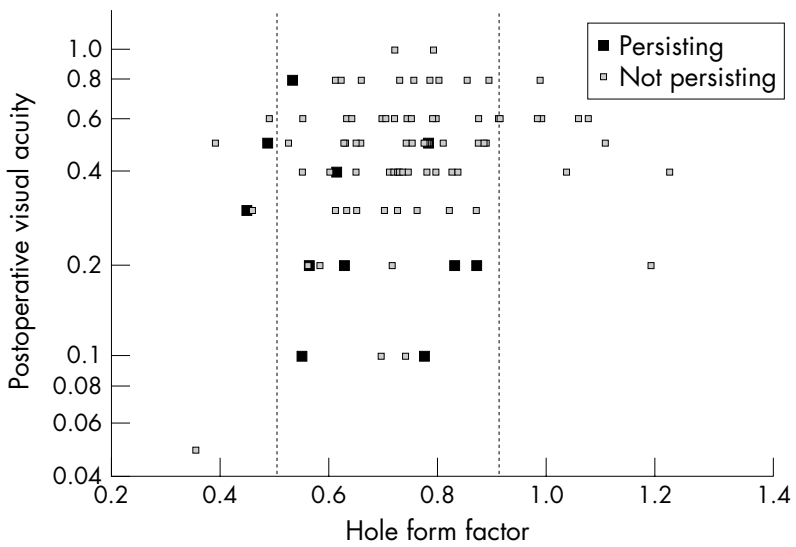

Figure 3 Correlation of hole form factor and best corrected postoperative visual acuity.

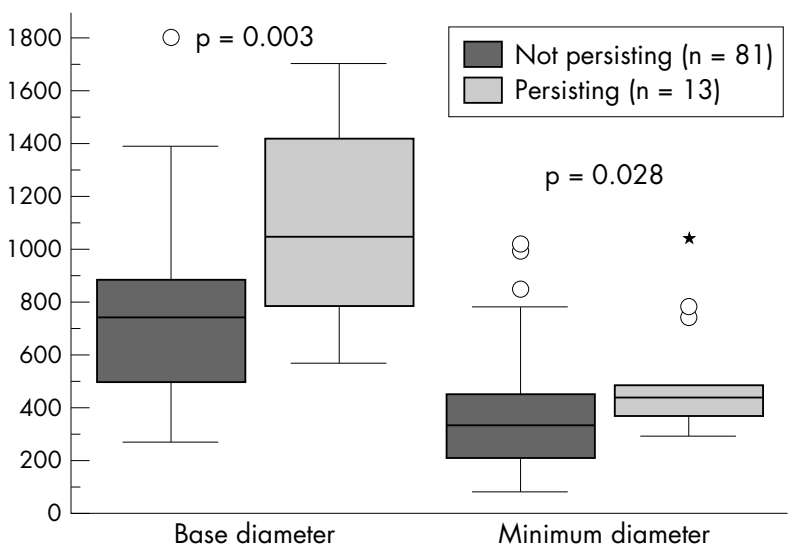

Figure 4 Base and minimum diameters of eyes with and without immediate macular hole closure.

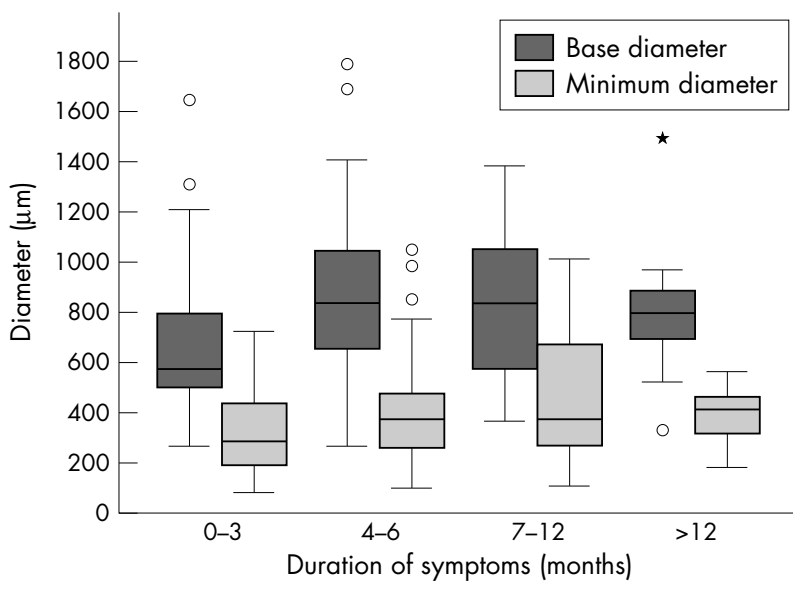

Figure 5 Correlation of the duration of symptoms and diameters of the macular hole.

evaluation of stage III and IV macular holes might be due to difficulties in detecting posterior vitreous detachment.

We also calculated the hole form factor (HFF), originally created by Puliafito. He considered the ratio between the overlying tissue dimensions and the hole base diameter to be of greater influence on the anatomical success rate than the base diameter alone. Puliafito found an $80 \%$ anatomical success rate in patients with HFF greater than 0.9 and an anatomical success rate of less than $25 \%$ in patients with HFF under
0.5. ${ }^{12}$ The association between HFF and anatomical success rate was found to be statistically significant. With respect to the correlation coefficient, $r$, the minimum diameter measured with OCT seemed to be a better predictor than the HFF.

In our study all patients with an HFF greater than 0.9 were operated on successfully in the first surgical approach and the anatomical success rate was $67 \%$ in patients with HFF under 0.5 .

Besides better anatomical results compared to those of Puliafito and colleagues, we also found a significant correlation between HFF and anatomical success rate. A possible explanation for the higher success rate in our patients compared with those of Puliafito et al might be that eventual existing epiretinal membranes including the inner limiting membrane were removed during surgery in our series.

Unexpectedly, the diameter of the macular hole measured with OCT was not influenced by the duration of symptoms. Therefore, large holes did not necessarily exist longer than small ones. This fact may be explained by our current understanding of the pathogenesis of idiopathic macular holes. The most favourable explanation for the development of a macular hole is traction caused by focal shrinkage of the prefoveolar vitreous. ${ }^{914}$ Also glial cells and newly formed collagen may play an important part in macular hole formation by exerting tangential traction. ${ }^{15}$ The diameter of the hole therefore may depend mainly on traction forces and not on the duration of the macular hole. Another reason for a missing correlation between the duration of symptoms and macular hole size may be the subjective estimation of the duration of symptoms by the patient. A macular hole may exist a long time before being detected. Therefore, our groups A-D may not reflect the real duration of the existence of the macular hole.

In conclusion, preoperative optical coherence tomography measurements seem to be of predictive value for the functional and anatomical outcome of macular hole surgery.

\section{Authors' affiliations \\ S Ullrich, C Haritoglou, C Gass, M Schaumberger, M W Ulbig, A Kampik, Department of Ophthalmology, \\ Ludwig-Maximilians-University, Munich, Germany \\ Financial interest: none. \\ Correspondence to: Professor Dr A Kampik, Department of Ophthalmology, Ludwig-Maximilians-University, Mathildenstrasse 8, D-80336 München, Germany \\ Anselm.Kampik@ak-i.med.uni-muenchen.de}

Accepted for publication 5 October 2001

\section{REFERENCES}

1 Noyes HD. Detachment of the retina, with laceration at the macula lutea. Trans Am Ophthalmol Soc 1871;1:128-9.

2 Kelly NE, Wendel RT. Vitreous surgery for idiopathic macular holes: results of a pilot study. Arch Ophthalmol 1991;109:654-9.

3 Margherio RR, Margherio AR, Williams GA, et al. Effect of perifoveal tissue dissection in the management of acute idiopathic full-thickness macular holes. Arch Ophthalmol 2000;1 18:495-8.

4 Park DW, Isperly JO, Sneed SR, et al. Macular hole surgery with internal-limiting membrane peeling and intravitreous air. Ophthalmology 1999:106:1392-8.

5 Mester U, Becker M. Prognosefaktoren bei der Makulachirurgie. Ophthalmologe 1998, 95:158-62.

6 Haritoglou C, Gass CA, Schaumberger M, et al. Macular changes after peeling of the internal limiting membrane in macular hole surgery. Am J Ophthalmol 2001;132:363-8.

7 Hee MR, Puliafito CA, Carlton W, et al. Optical coherence tomography of macular holes. Ophthalmology 1995;102:748-56.

8 Baumal CR. Clinical application of optical coherence tomography. Curr Opin Ophthalmol 1999;10:182-8.

9 Gass JDM. Idiopathic senile macular hole; its early stages and pathogenesis. Arch Ophthalmol 1988;106:629-39. 
10 Tornambe PE, Poliner LS, Grote K. Macular hole surgery without face-down positioning. Retina 1997;17:179-85.

11 Wendel RT, Patel AC, Kelly NE, et al. Vitreous surgery for macular holes. Ophthalmology 1993;100:1671-6.

12 Desai VN, Hee MR, Puliafito CA. Optical coherence tomography of macular holes. In: Madreperla SA, McCuen BW, eds. Macular hole: pathogenesis, diagnosis and treatment. Oxford: Butterworth-Heinemann 1999:37-47.
13 Freeman WR, Azen SP, Kim JW, et al. Bailey I for the vitrectomy for treatment of macular hole study group. Vitrectomy for the treatment of treatment of macular hole study group. Vitrectomy for the treat
full-thickness stage 3 or 4 macular holes. Arch Ophthalmol full-thickness stage

14 Margherio RR, Schepens CL. Macular breaks. 1. Diagnosis, etiology and observations. Am J Ophthalmol 1972;74:219-32.

15 Messmer EM, Heidenkummer HP, Kampik A. Ultrastructure of epiretinal membranes associated with macular holes. Graefes Arch Clin Exp Ophthalmol 1998;236:248-54.

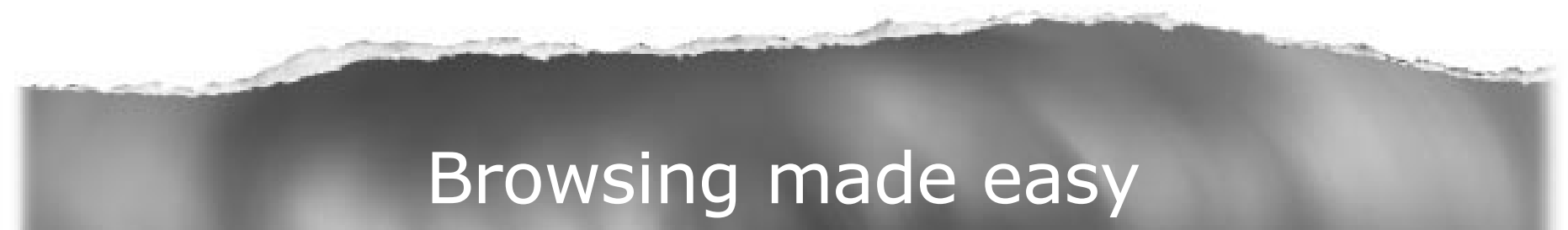

\section{Collections}

With a single click Collections allows you to find all articles that have been published in your chosen subject. Select from over 200 clinical and non-clinical topic collections and/or cross search other specialist journals, the BMJ and Cochrane Reviews

www.bjophthalmol.com 